\title{
Desplazamientos de la iguana verde, Iguana iguana (Squamata: Iguanidae) durante la estación seca en La Palma, Veracruz, México
}

\author{
Jorge E. Morales-Mávil ${ }^{1}$, Richard C. Vogt ${ }^{2} \&$ Héctor Gadsden-Esparza ${ }^{3}$ \\ 1 Instituto de Neuroetología, Universidad Veracruzana. Ap. Postal 566, Xalapa, Veracruz, México. Fax: (228) 8418920; \\ mmavil@correoneuro.net \\ 2 Instituto Nacional de Pesquisas da Amazônia, Caxia Post. 478, Manaus, Amazonas, Brasil cp. 69083-000; vogt@inpa. \\ gov.br \\ 3 Instituto de Ecología, A.C., Centro Regional Chihuahua, Km. 33.3, carretera Chihuahua-Ojinaga, Cd. Aldama, Chich., \\ México, Ap. Postal 28; hector.gadsden@inecol.edu.mx
}

Recibido 29-X-2003. Corregido 24-VIII-2006. Aceptado 28-XI-2006.

\begin{abstract}
Displacements of the green iguana (Iguana iguana) (Squamata: Iguanidae) during the dry season in La Palma, Veracruz, Mexico. The green iguana (Iguana iguana) is said to be primarily sedentary, although the females travel long distances to nest. Displacement patterns must be known to help predict the effects of environmental disturbance on iguanas' survival. We studied nesting season (February-July) movements in La Palma, Los Tuxtlas, Veracruz, Mexico (18 $33^{\prime}$ N, $\left.95^{\circ} 03^{\prime} \mathrm{W}\right)$. Individual movements and activity were monitored by radio tracking. The transmitters were implanted surgically in eight adult iguanas (four males and four females). Snout vent length (SVL) was used to determine the relationship between size of the body and size of home range. To estimate the size of home range, three or more points were used. Minimum convex polygons estimates of home range were calculated with McPAAL. The iguanas were radio-located between 23 and 30 occasions, mainly in trees (56\% between 3-9 m); only $4 \%$ were localized under a height of $3 \mathrm{~m}$ (forest floor). The occupation area mean was larger for males (9 158.06 $\left.\pm 3025.3 \mathrm{~m}^{2} \mathrm{vs} .6591 .24 \pm 4001.1 \mathrm{~m}^{2}\right)$ although the differences were not significant $(\mathrm{t}=0.51, \mathrm{p}>0.05)$. SVL was correlated with home range $(\mathrm{r}=0.76 ; \mathrm{gl}=7$; $\mathrm{p}<0.05$ ). Breeding males defended their home range vigorously against other adult males. We observed one separate male home range and large portions of overlap between the sexes. The home range generally formed a conglomerate of polygons and only two had linear shapes along the river: apparently iguanas use the riparian vegetation for foraging. The females display two strategies for nesting: 1) moving to the sandy area near the sea or, 2) laying eggs near the river, in loam. Iguanas responded to habitat fragmentation and reduction by modifying their nesting strategy. Rev. Biol. Trop. 55 (2): 709-715. Epub 2007 June, 29.
\end{abstract}

Key words: home ranges, green iguana, Los Tuxtlas, nesting, radiotracking.

La iguana verde (Iguana iguana) ha sido considerada principalmente sedentaria, aunque se conoce que las hembras realizan largos recorridos para llegar a las playas arenosas para anidar comunalmente (Montgomery et al. 1973, Bock et al. 1989, Rand et al. 1989). No obstante, existen sitios donde algunas iguanas (Cyclura) llegan a anidar en suelos menos accesibles para la construcción de nidos (Wiewandt 1979). Cuando esto ocurre, las iguanas seguramente se enfrentan a conflictos de supervivencia que pueden estar relacionados con asegurar el éxito en la anidación, incubación y eclosión, viajando a los sitios arenosos; o permanecer en su ámbito hogareño, sin la necesidad de trasladarse grandes distancias por zonas poco conocidas.

La extensión y la forma del ámbito hogareño $(\mathrm{AH})$, están relacionadas con los factores ambientales que ejercen presión selectiva sobre las especies, principalmente la disponibilidad de recursos. La estimación del área que cubre el ámbito hogareño, no es un indicador suficiente de lo que se encuentra en su interior (Curtis y Zaramody 1998). Por esto, es necesario incluir 
otros parámetros que ayuden a interpretar lo que está ocurriendo, uno de ellos es el conocer el patrón de desplazamientos que hacen los animales en el interior del ámbito hogareño, que al relacionarlo con la forma y extensión del $\mathrm{AH}$, nos da una mayor idea del modo en que son utilizados los recursos.

Es necesario conocer los patrones de movimiento y el uso del espacio para comprender mejor la historia natural de las iguanas. Particularmente, conocer el uso del ámbito hogareño es importante para predecir los efectos del disturbio ambiental y la fragmentación del ambiente sobre la supervivencia de las iguanas. Este trabajo explora el desplazamiento durante el periodo de anidación (febrero-julio) de iguanas verdes adultas, con el objetivo de conocer si las hembras que habitan zonas fragmentadas y perturbadas a no más de $3 \mathrm{~km}$ de distancia de la playa, viajan a depositar sus huevos en las zonas arenosas o deciden anidar en lugares menos apropiados.

\section{MATERIALES Y MÉTODOS}

El trabajo fue realizado en La Palma, región de Los Tuxtlas, Veracruz, México $\left(18^{\circ} 33^{\prime} \mathrm{N}\right.$, $95^{\circ} 03^{\prime} \mathrm{W}$ ) (Fig. 1). El área tiene una altitud entre 50 y $230 \mathrm{msnm}$. La precipitación media anual es de $4900 \mathrm{~mm}$, con una temporada de lluvias entre junio y noviembre. El tipo de vegetación predominante en la zona es la selva alta perennifolia, aunque con fuerte grado de perturbación, y en los márgenes de los ríos se localiza vegetación ribereña. Existe también, a manera de pequeños remanentes situados en las partes de topografía accidentada, selva mediana perennifolia, y en las partes bajas, cercanas a la porción costera, manglar y vegetación de dunas costeras (playa arenosa) (Ibarra-Manríquez et al. 1997). El río La Palma, con varios afluentes, cruza el área de estudio.

Los movimientos individuales y las áreas de actividad de las iguanas fueron registrados con radiotelemetría. Se colocaron quirúrgicamente transmisores (diseñados por AVM Instrument Company) en ocho iguanas adultas (cuatro machos y cuatro hembras). Los transmisores tuvieron un peso entre 10-13 g, y fueron insertados bajo la piel en la parte dorso-lateral de cada iguana. Cada transmisor producía un pulso continuo en una frecuencia única para cada individuo. Las iguanas fueron liberadas al siguiente día de la cirugía. Cada individuo se marcó por inyección de microchips (pet-iD, FDXB) conteniendo un registro numérico electrónico; este marcaje funcionó como alternativa para obtener información al recapturarlos, en caso de que a algún individuo se le llegase a desprender el radiotransmisor. También se utilizaron marcas temporales, pintando con tinta

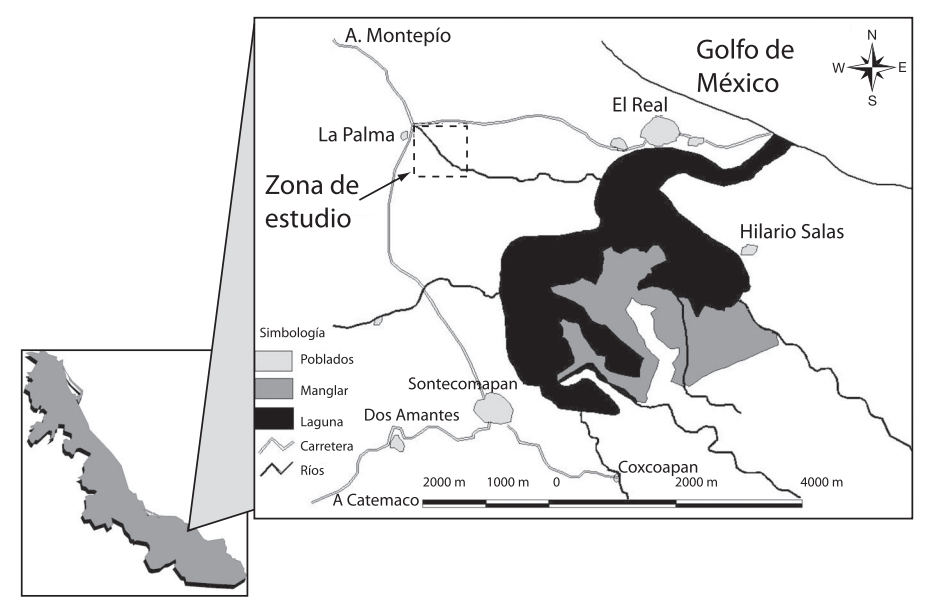

Fig.1. Lugar del estudio: la Palma, Los Tuxtlas, Veracruz, México.

Fig.1. Study site: La Palma, Los Tuxtlas, Veracruz, Mexico. 
indeleble a los individuos en el dorso, con una clave numérica de aproximadamente $2 \mathrm{~cm}$ de longitud, de esta manera se facilitó la observación a distancia.

Las radiolocalizaciones fueron efectuadas entre las 08:00 y las 18:00 h (dos o tres veces por semana de febrero a julio de 1999), debido a las limitaciones en el tiempo útil de los transmisores (un año máximo); éstos podían ser detectados a una distancia aproximada de $500 \mathrm{~m}$. Los animales se detectaron a distancia, marcándose sólo las coordenadas del árbol donde se observaron y estimando, con ayuda de un clinómetro, la altura a la que se encontraban, con la finalidad de conocer los estratos de mayor uso.

Cada punto en el que se localizó el individuo rastreado, fue marcado con una placa de aluminio numerada, fechada y fijada al tronco del árbol. Se midió la distancia de desplazamiento de la última localización al nuevo punto de registro y se obtuvieron las características ambientales (temperatura, porcentaje de humedad y altura en la que se detectó el individuo) de todas las localizaciones subsecuentes; cada una de éstas fue apuntada en un mapa base del área de estudio. Se utilizó un GPS (Garmin $12 \mathrm{XL}$ ) para obtener las coordenadas X y Y (UTM) de cada localización de las iguanas.
La longitud hocico-cloaca (LHC, en $\mathrm{mm}$ ) se utilizó para correlacionar el tamaño del cuerpo y el tamaño del ámbito hogareño usando el coeficiente de correlación de Pearson. Para calcular el tamaño del ámbito hogareño se utilizó el programa McPAAL (Stüwe y Blohowiak 1985) por el método de polígonos convexos (PC) no ajustados (Southwood 1966) utilizando tres o más puntos separados de recaptura por individuo.

\section{RESULTADOS}

Las iguanas radiolocalizadas fueron observadas principalmente sobre los árboles $(95.67$ $\%$ de los registros): $18.6 \%$ entre $12-15 \mathrm{~m}$, $20.8 \%$ entre $9-12 \mathrm{~m}, 29.4 \%$ entre $6-9 \mathrm{~m}$ y $26.8 \%$ entre 3-6 m de altura. Sólo el $4.3 \%$ se localizaron por debajo de los $3 \mathrm{~m}$ de altura, básicamente sobre el piso de la selva. Todos los individuos permanecieron cerca de la vegetación ribereña y de la selva perturbada y una hembra (HE3) viajó hacia la playa arenosa.

En el Cuadro 1 se muestran las estimaciones del ámbito hogareño, incluyendo los movimientos de la iguana HE3 que viajó a depositar sus huevos a la playa. Perdimos contacto por varios días con este individuo y

CUADRO 1

Áreas de actividad durante la estación seca, de ocho individuos de iguana verde (Iguana iguana)

TABLE 1

Dry season activity areas for eight adult iguanas (Iguana iguana)

$\begin{array}{ccccc}\text { Sexo } & \text { Individuos } & \text { LHC }(\mathrm{mm}) & \text { Número de localizaciones } & \text { Ámbito hogareño }\left(\mathrm{m}^{2}\right) \\ \text { Macho } & \text { MA1 } & 350 & 30 & 7122.0 \\ \text { Macho } & \text { MA2 } & 470 & 30 & 14163.1 \\ \text { Macho } & \text { MA3 } & 385 & 30 & 1500.0 \\ \text { Macho } & \text { MA4 } & 440 & 28 & 13847.1 \\ \text { Hembra } & \text { HE1 } & 375 & 30 & 4606.7 \\ \text { Hembra } & \text { HE2 } & 279 & 30 & 1148.3 \\ \text { Hembra } & \text { HE3 } & 405 & 23 & 18397.0 \\ \text { Hembra } & \text { HE4 } & 290 & 30 & 2213.0\end{array}$


no fue posible localizar el sitio donde depositó sus huevos, pese a que el radiotransmisor permaneció adherido. Es probable que la distancia para la recepción no fue la adecuada o las condiciones ambientales no permitieron captar la señal, posiblemente influyó su deambular entre espesa vegetación o dentro del agua.

Los resultados mostraron una gran variabilidad en las áreas de desplazamiento globales de los ocho individuos $(\mathrm{x} \pm \mathrm{ES}=7874.6 \pm 2372.1$ $\mathrm{m}^{2}$; intervalo 1 148.3-1 $8397 \mathrm{~m}^{2}$ ). Sin embargo, el promedio del tamaño de los ámbitos hogareños de los machos y de las hembras fue similar (9 158.06 $\pm 3025.3 \mathrm{~m}^{2}$ vs. $6591.24 \pm 4001.1 \mathrm{~m}^{2}$ ), respectivamente; $(\mathrm{t}=0.51, \mathrm{p}>0.05)$, debido, posiblemente, al tamaño de muestra reducido. Se encontró una correlación positiva entre el tamaño de los ámbitos hogareños y la LHC de los organismos $(\mathrm{r}=0.76 ; \mathrm{gl}=7 ; \mathrm{p}<0.05)$.

Los animales se localizaron básicamente en la vegetación ribereña y en la zona anexa a ésta; aunque llegaron a ubicarse en áreas abiertas (pastizal-acahual), generalmente fueron observados en hábitat con abundante vegetación arbustiva y arbórea. Un macho (MA4) y una hembra (HE3), tuvieron más desplazamiento lineal a lo largo del río, que el resto de los individuos, los cuales se agruparon en la zona selvática formando una aglomeración de polígonos. El macho MA2, el de mayor tamaño $(\mathrm{LHC}=470 \mathrm{~mm})$, presentó mayor actividad de locomoción (Fig. 2). El área de desplazamiento del MA4 no se sobrepuso con el conglomerado de polígonos que formaron los ámbitos hogareños de los individuos MA1, MA2, MA3, HE1, H2 y H4, sólo se traslapó con el ámbito hogareño de la HE3, la cual realizó desplazamiento básicamente por la vegetación ribereña, con dirección a la playa arenosa de la Barra de Sontecomapan.

Durante los muestreos, las iguanas fueron localizadas principalmente asoleándose y alimentándose. Hojas y frutos de Ficus tecolutensis, Coccoloba hondurensis, Pimenta dioica y Eugenia capuli, fueron consumidas por los individuos observados. En seis ocasiones se observó que los machos adultos defendieron su territorio vigorosamente contra otros machos. De manera general, esta defensa se asoció a un comportamiento de intenso movimiento de cabeza y erección de la papada y de la cresta vertebral.

Se registró una gran proporción de sobreposiciones entre los polígonos obtenidos para los dos sexos; también puede observarse el ámbito hogareño de una hembra separado del resto y sobrepuesto sólo con el de un macho. Siete de los polígonos cubren principalmente los sitios cercanos al río (Fig. 2). Más del $60 \%$ de las iguanas recapturadas y observadas a menos de $200 \mathrm{~m}$ de su primera recaptura, proporciona una estimación del alto grado de sedentaridad de la especie. El conjunto de polígonos refleja, en general, ámbitos hogareños fijos para los individuos muestreados.

Las iguanas hembras de la zona de $\mathrm{La}$ Palma desplegaron dos estrategias para anidar: 1) o viajaron a las áreas arenosas de la playa, aunque considerando que esta conducta sólo fue realizada por un individuo, 2) o permanecieron en su hábitat ripario.

\section{DISCUSIÓN}

El tamaño de los ámbitos hogareños de las iguanas en Los Tuxtlas es similar a los descritos por Bock et al. (1989) y Rand et al. (1989) en Panamá. Rand et al. (1989) usaron métodos semejantes a los nuestros y un tamaño de muestra menor (cinco individuos adultos, tres machos y dos hembras). Encontraron un ámbito hogareño para los adultos similar a los obtenidos en este estudio (9 $605.2 \pm 6 \quad 834.94$

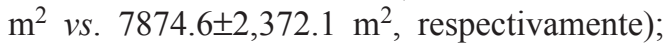
siendo para los machos de $14143.67 \pm 3869.87$ $\mathrm{m}^{2}$ (9 $158.06 \pm 3 \quad 025.3 \mathrm{~m}^{2}$ en este estudio) y para las hembras de $2797.5 \pm 1559.17 \mathrm{~m}^{2}$ (6 $591.24 \pm 4001.1 \mathrm{~m}^{2}$ en este estudio). Los movimientos mayores de los adultos estuvieron relacionados con la actividad reproductora. Las hembras se movieron grandes distancias durante el periodo de anidación; hasta más de $3 \mathrm{~km}$ para depositar sus huevos en la playa (Rand et al. 1989). Los resultados en este estudio muestran nuevas apreciaciones respecto a los movimientos de la iguana verde 


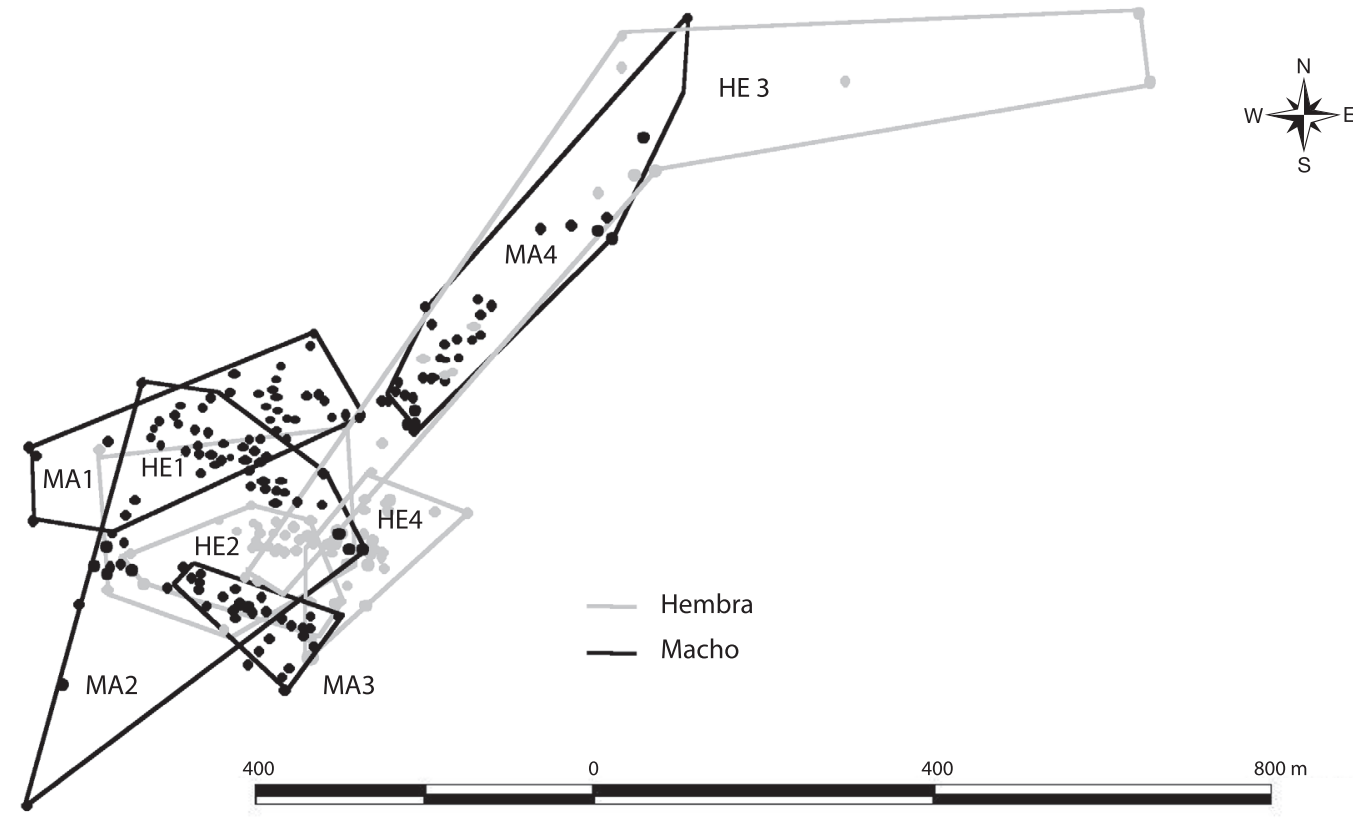

Fig. 2. Polígonos mínimos convexos del ámbito hogareño de ocho iguanas adultas (Iguana iguana).

Fig. 2. Minimal convex polygons that represent the home range of eight adult iguanas (Iguana iguana).

durante su periodo de reproducción, ya que se conocía como hecho generalizado para la región de Los Tuxtlas, que las iguanas eran sedentarias entre octubre y marzo; mientras que en abril las hembras viajaban más de 2 km para anidar hacia la playa (Garza y Vogt 1994). Sin embargo, esta conjetura solamente estaba basada en el registro de la distancia de desplazamiento de aproximadamente $2.5 \mathrm{~km}$ de dos hembras que viajaron hacia las playas de Jicacal. Aparentemente, este comportamiento se da, sólo cuando las hembras encuentran en su recorrido hacia la playa, la cobertura vegetal adecuada para su protección.

Aunque no fue observada la anidación, se tuvo el registro de que al menos dos de las hembras grávidas no viajaron a la playa y depositaron sus huevos cerca del río. El comportamiento de anidar en lugares sin suelo arenoso ha sido observado para algunas especies de iguanas del género Cyclura (Carpenter 1967, Wiewandt 1982, Vogel 1994, 2000, Wiewandt y García 2000). Ellos encontraron que algunas hembras de este grupo de iguanas no viajan a la costa para anidar, sino que frecuentemente depositan sus huevos cerca de la ribera en tierra arcillosa o en huecos de zonas pedregosas. Nosotros pensamos que este fenómeno se presenta en la iguana verde cuando la distancia de desplazamiento hacia los sitios de anidación es muy grande, cuando la presión de los depredadores es alta o cuando existe competencia por los lugares para anidar; es entonces cuando muchas hembras, principalmente las jóvenes deciden permanecer cerca de sus centros de actividad.

Los tamaños del ámbito hogareño observados en este estudio, fueron poco mayores a los informados por Rand et al. (1989) en Barro Colorado, las características del hábitat podrían ser un factor importante que afecte este tamaño. La Palma tiene condiciones desfavorables con respecto a las áreas mejor conservadas de la Isla Barro Colorado en Panamá, es decir, existe un bosque perturbado por plantaciones de pimienta y naranjo, rodeadas por grandes áreas abiertas a la ganadería, lo cual contrasta con la selva perennifolia en buen estado que se menciona para Barro Colorado. El paisaje fragmentado y perturbado de La Palma, podría influir 
en que las iguanas tengan la necesidad de realizar mayores desplazamientos para encontrar recursos alimentarios suficientes, principalmente frutos. En ese sentido, se conoce que las iguanas verdes de La Palma, incluyen frutos en su dieta durante todo el año y muchas de las especies que utilizan para obtener este recurso se encuentran en números reducidos y con individuos aislados en la zona.

Es probable que el área de actividad determinada para la HE3 (18 $397 \mathrm{~m}^{2}$ ), esté influyendo para que se consideren similares los ámbitos hogareños de machos y hembras, ya que el desplazamiento particular de este individuo, provoca un incremento en el promedio determinado para las hembras. Para que esto no ocurra, quizá deberíamos considerar eliminar la notable sección del desplazamiento de este individuo fuera del sitio de mayor agrupación del resto de las iguanas radiolocalizadas, tal y como lo sugieren Rand et al. (1989). Este estudio fue realizado durante la estación seca del año; para entender más el desplazamiento de las iguanas, será necesario complementar el registro durante la estación húmeda. Durante el periodo de lluvias, las iguanas podrán obtener mayor cantidad de recursos alimentarios y refugio por el incremento en la cobertura del ambiente, con lo cual posiblemente variará su comportamiento $\mathrm{y}$, en particular, su ámbito hogareño.

Las iguanas hembras de la zona de $\mathrm{La}$ Palma despliegan por lo menos dos estrategias para anidar: 1) viajan a las áreas arenosas de las playas 0,2$)$ permanecen en el hábitat ripario. La primera tiene la ventaja de que las hembras llegan a una zona con el suelo óptimo para anidar, donde pueden construir sus elaborados nidos para proteger sus huevos y con esto asegurar altas temperaturas para la incubación y la eclosión de su progenie. Sin embargo, presenta algunas desventajas, por ejemplo, el viajar a través de un ambiente drásticamente alterado, carente de vegetación arbórea en parte de su trayecto, eleva el riesgo de depredación, principalmente por la gente. Además, la anidación que realizan es comunal, por lo tanto, las iguanas deberán competir entre sí por encontrar los mejores sitios para anidar. Otro inconveniente es que, al nacer las crías, éstas, para llegar a un área con vegetación suficiente para obtener recursos alimentarios y cobijo, deberán viajar una distancia similar a la realizada por las hembras, debido a que se trata de un ambiente fragmentado, pero con mayores desventajas, ya que pueden ser presa de un mayor número de depredadores, incluyendo aves, mamíferos y serpientes.

La segunda alternativa de anidación (permanecer en su ambiente ripario) tiene la ventaja de no requerir que las hembras se desplacen grandes distancias para anidar, por lo tanto, la exposición de éstas hacia los depredadores es baja, ya que se mantienen dentro del hábitat que reconocen. Sin embargo, el nido que llegan a construir es muy superficial, debido a la dureza del terreno; además, la baja radiación solar que llega al suelo, producto de una densa cobertura vegetal, no provee una temperatura adecuada para la incubación. Estos inconvenientes ambientales provocan que exista una alta depredación de nidadas por parte de mapaches, tlacuaches y humanos (obs. pers.), y que el éxito de eclosión sea bajo. Cualquiera de las dos alternativas de anidación, dejan entrever graves problemas para la supervivencia de las crías.

La población de iguanas está restringida en pequeños grupos distribuidos en un sistema de fragmentos de selva. Por lo tanto, la información aquí obtenida, puede reflejar los problemas que enfrentan estos grupos de iguana verde que se encuentran en ambientes fragmentados de varias localidades de Veracruz y del sudeste mexicano. El paisaje donde habitan las iguanas ha influido para que existan cambios en la estrategia de anidación de este reptil en la búsqueda para lograr así su permanencia dentro de estos ambientes fragmentados.

\section{AGRADECIMIENTOS}

Al Consejo Nacional de Ciencia y Tecnología (CONACYT, registro 48179) y a la Dirección General de Estudios de Postgrado de la UNAM (DGEP), por las becas otorgadas al primer autor. Al Patronato Pro-Universidad 
Veracruzana, A.C., por el apoyo otorgado para la realización del trabajo. A Gonzalo Pérez Higareda, Laura, E. Domínguez Domínguez y Margarita Garza Castro, por las sugerencias al manuscrito. A Paulo César Quintana Morales por su apoyo cartográfico y a Marco Antonio López Luna y Felipe Delfín Limón, por su apoyo en el trabajo de campo.

\section{RESUMEN}

Usamos radiotransmisores para determinar los desplazamientos de la iguana verde (Iguana iguana) en el periodo de anidación (febrero-julio) en La Palma, Los Tuxtlas, Veracruz, México (18³3’ N, 9503’ W). Las iguanas fueron radiolocalizadas entre 23 y 30 ocasiones, principalmente en árboles (56\% entre 3-9 m); sólo $4 \%$ fueron localizadas en el suelo. El tamaño del ámbito hogareño de machos y de hembras fue similar (9 $158.06 \pm 3025.3 \mathrm{~m}^{2} \mathrm{vs}$. $6591.24 \pm 4001.1 \mathrm{~m}^{2}$, respectivamente; $\left.\mathrm{t}=0.51, \mathrm{p}>0.05\right)$. Se encontró una correlación significativa entre la LHC y el ámbito hogareño $(r=0.76, g l=7, p<0.05)$. Las hembras tienen dos estrategias para anidar: 1) viajan para depositar los huevos a la arena de la playa, o 2) permanecen cerca de su ambiente ribereño y anidan en suelo arenoso-arcilloso. Al parecer, el ambiente fragmentado de la zona de la Palma, influye en la estrategia de anidación de la iguana verde.

Palabras clave: ámbito hogareño, iguana verde, Los Tuxtlas, anidación, radiotelemetría.

\section{REFERENCIAS}

Bock, B.C., S. Rand \& G.M. Burghardt. 1989. Nesting season movements of female green iguanas (Iguana iguana) in Panama. Copeia 1989: 210-212.

Carpenter, C.C. 1967. Aggression and social structure in iguanid lizards, p. 87-105. In W.W Milstead (ed.). Lizard ecology: A symposium. Columbia, Missouri, EEUU.

Curtis, D.J. \& A. Zaramody. 1998. Group size, home range use and seasonal variation in the ecology of Eulemur mongoz. Int. J. Primat. 19-5: 811-835.
Garza, C.M. \& R.C. Vogt. 1994. Algunos aspectos del manejo de la iguana verde (Iguana iguana) en Los Tuxtlas, Veracruz, México. In III Reunión Nacional de Herpetología, Sociedad Herpetológica Mexicana, Chiapas, México.

Ibarra-Manríquez, G., M. Martínez-Ramos, R. Dirzo \& J. Núñez-Farfán. 1997. La Vegetación, p. 61-82. In E. González-Soriano R. Dirzo \& R.C. Vogt (eds.). Historia Natural de Los Tuxtlas. UNAM y Conabio, México DF, México.

Montgomery, G.G., A.S. Rand \& M.E. Sundquist. 1973. Postnesting movements of iguanas from a nesting aggregation. Copeia 1973: 620-622.

Rand, A.S., E. Font, D. Ramos, D.I. Werner \& B.C. Bock. 1989. Home range in green iguanas (Iguana iguana) in Panama. Copeia 1989: 217-221.

Southwood, R.E. 1966. Ecological methods. Methuen, Londres, Inglaterra.

Stüwe, M. \& C.E. Blohowiak. 1985. Micro-computer programs for the analysis of animal locations (MACPAAL, version 1.2). Smithsonian Institution, Washington DC, EEUU.

Vogel, P. 1994. Evidence of reproduction in a remnant population of the endangered Jamaican iguana, Cyclura collei (Lacertilia: Iguanidae). Caribbean J. Sci. 30:234-241.

Vogel, P. 2000. Jamaican iguana Cyclura collie, p. 19-22. In A. Alberts (ed.). West Indian Iguanas: Status Survey and Conservation Action Plan. IUCN, Londres, Inglaterra.

Wiewandt, T.A. 1979. La gran iguana de Mona. Natur. Histor. 88: 56-65.

Wiewandt, T.A. 1982. Evolution of nesting patterns in Iguanine lizards, p. 119-141. In G.M Burghardt \& A.S. Rand (eds.). Iguanas of the world. Their behavior, ecology and conservation. Noyes, Nueva Jersey, EEUU.

Wiewandt, T.A. \& M. García. 2000. Mona Island iguana Cyclura cornuta stejnegeri, p. 27-31. In A. Alberts (ed.). West Indian Iguanas: Status Survey and Conservation Action Plan. IUCN, Londres, Inglaterra. 
